\title{
Antitumor activities of JTP-74057 (GSK1120212), a novel MEK1/2 inhibitor, on colorectal cancer cell lines in vitro and in vivo
}

\author{
TAKAYUKI YAMAGUCHI ${ }^{1}$, REINA KAKEFUDA ${ }^{1}$, NOBUYUKI TAJIMA ${ }^{1}$, \\ YOSHIHIRO SOWA $^{2}$ and TOSHIYUKI SAKAI ${ }^{2}$ \\ ${ }^{1}$ Central Pharmaceutical Research Institute, Japan Tobacco Inc., Takatsuki, Osaka 569-1125; \\ ${ }^{2}$ Department of Molecular-Targeting Cancer Prevention, Graduate School of Medical \\ Science, Kyoto Prefectural University of Medicine, Kyoto 602-8566, Japan
}

Received January 21, 2011; Accepted March 18, 2011

DOI: $10.3892 /$ ijo.2011.1015

\begin{abstract}
The MAPK pathway is one of the most important pathways for novel anticancer drug development. We performed high-throughput screening for compounds that induce expression of p15 $5^{\text {INK4b }}$, and identified JTP-74057 (GSK1120212), which is being evaluated in ongoing phase I, II and III clinical trials. We characterized its antitumor activities in vitro and in vivo. JTP-74057 strongly inhibited MEK1/2 kinase activities, but did not inhibit another 98 kinase activities. Treatment by JTP-74057 resulted in growth inhibition accompanied with upregulation of $\mathrm{p} 15^{\text {INK4b }}$ and/or $\mathrm{p} 27^{\mathrm{KIP1}}$ in most of the colorectal cancer cell lines tested. Daily oral administration of JTP-74057 for 14 days suppressed tumor growth of HT-29 and COLO205 xenografts in nude mice. Notably, tumor regression was observed only in COLO205 xenografts, and COLO205 was much more sensitive to JTP-74057-induced apoptosis than HT-29 in vitro. Treatment with an Akt inhibitor enhanced the JTP-74057-induced apoptosis in HT-29 cells. Finally, JTP-74057 exhibited an additive or a synergistic effect in combination with the standard-of-care agents, 5-fluorouracil, oxaliplatin or SN-38. JTP-74057, a highly specific and potent MEK1/2 inhibitor, exerts favorable antitumor activities in vitro and in vivo. Sensitivity to JTP-74057-induced apoptosis may be an important factor for the estimation of in vivo efficacy, and sensitivity was enhanced by an Akt inhibitor. These results suggest the usefulness of JTP-74057 in therapeutic applications for colorectal cancer patients.
\end{abstract}

Correspondence to: Toshiyuki Sakai, Department of MolecularTargeting Cancer Prevention, Graduate School of Medical Science, Kyoto Prefectural University of Medicine, Kawaramachi-Hirokoji, Kamigyo-ku, Kyoto 602-8566, Japan

E-mail: tsakai@koto.kpu-m.ac.jp

Takayuki Yamaguchi, Central Pharmaceutical Research Institute, Japan Tobacco Inc., 1-1 Murasaki-cho, Takatsuki, Osaka 569-1125 E-mail: takayuki.yamaguchi@jt.com

Key words: MEK, JTP-74057, GSK1120212, antitumor activity, PI3K/Akt

\section{Introduction}

The MAPK pathway is a central signal transduction pathway of cell proliferation and survival $(1,2)$. Various growth factors bind to their receptor tyrosine kinases on the cell surface and induce dimerization and autophosphorylation of the receptors followed by association of adaptor proteins. Subsequently, the guanine nucleotide exchange factor Sos activates Ras by catalyzing the replacement of GDP with GTP. GTP-bound Ras then recruits the serine/threonine kinase Raf (A-Raf, B-Raf or c-Raf) to the plasma membrane where it becomes activated, resulting in phosphorylation of MEK1/2 by the Raf kinase. Activated MEK1/2 in turn phosphorylate ERK1/2 at specific threonine and tyrosine residues. The phosphorylated ERK1/2 phosphorylate and activate a variety of substrates, such as p $90^{\mathrm{RSK} 1}$. Also, ERK1/2 themselves can translocate to the nucleus and induce transcription of genes for cell proliferation and survival. In human malignant tumors, the Raf-MEK-ERK pathway is often hyperactivated through aberrant activation of the receptor tyrosine kinases or gain-of-function mutations in the Ras or B-Raf gene (1). One of the Ras gene family proteins, $\mathrm{K}$-Ras, is reported to be mutated in $90 \%$ of pancreatic cancers, $45 \%$ of colorectal cancers and $35 \%$ of non-small cell lung cancers $(3,4)$. B-Raf mutations have been identified in $66 \%$ of melanomas, $12 \%$ of colorectal cancers and $35-70 \%$ of papillary thyroid cancers (5-9). The overexpression and/or mutation of epidermal growth factor (EGF) receptor (EGFR), erbB2, platelet-derived growth factor receptor and other growth factor receptors have also been observed in many types of cancers $(10,11)$. Thus, a component of the Raf-MEK-ERK pathway has been considered as an attractive target for a novel approach to chemotherapy. CI-1040 (also called PD184352) was the first MEK1/2 inhibitor evaluated in clinical trials $(12,13)$, although its clinical development was discontinued due to insufficient efficacy. The second generation MEK1/2 inhibitors, PD0325901 and AZD6244 (ARRY-142886), which have shown better efficacy than CI-1040 in preclinical models, have also been tested in clinical trials $(1,2,14,15)$.

Based on our original screening strategy for moleculartargeting agents, we initiated a high-throughput screening for compounds that induce expression of cyclin-dependent kinase (CDK) inhibitors, such as $15^{\mathrm{INK} 4 \mathrm{~b}}, \mathrm{p} 21^{\mathrm{WAF} 1}$ or $\mathrm{p} 27^{\mathrm{KIP} 1}$. Notably, 
we and others found that several molecular-targeting agents against cancer, such as HDAC inhibitors, gefitinib, imatinib, or trastuzumab enhanced expression of CDK inhibitors (16-19), and we identified several novel molecular-targeting agents for clinical use by the screening vice versa (20), suggesting that our screening methods are useful for the development of novel molecular-targeting drugs for cancer. Based on this strategy, we conducted a high-throughput screening for compounds that induce the expression of $\mathrm{p} 15^{\mathrm{INK} 4 \mathrm{~b}}$, an endogenous inhibitory protein of CDK4/6, and found a pyridoprymidine derivative JTP-70902 (21). Subsequent analysis revealed that JTP-70902 is a novel MEK1/2 inhibitor, which has comparable efficacy to AZD6244 (14,21). Through the further efforts of medicinal chemistry, we discovered JTP-74057 [its dimethyl sulfoxide (DMSO)-solvate form is also called GSK1120212], which has the highest MEK1/2 inhibitory activity within the series. JTP-74057 showed much more potent antitumor activity than the second generation MEK inhibitors, such as PD0325901 or AZD6244, in vivo and is being evaluated in phase III clinical trial in melanoma patients with B-Raf mutations. Here, we report the biological profile of JTP-74057 and discuss its antitumor effects on human colorectal cancer cell lines in vitro and in vivo.

\section{Materials and methods}

Chemicals and reagents. JTP-74057, $N$-\{3-[3-cyclopropyl5-(2-fluoro-4-iodophenylamino)-6,8-dimethyl-2,4,7-trioxo3,4,6,7-tetrahydro-2 $H$-pyrido[4,3- $d$ ]pyrimidin-1-yl]phenyl\} acetamide and SN-38 were synthesized at Japan Tobacco Inc. (Osaka, Japan). API-2 was purchased from Calbiochem (Darmstadt, Germany). 5-Fluorouracil (5-FU) and oxaliplatin were purchased from Wako Pure Chemical Industries (Osaka, Japan) or Enzo Life Sciences (Farmingdale, NY), respectively. A vehicle of $0.1 \%$ DMSO was used for test compounds in vitro. Antibodies against $\mathrm{p} 15^{\mathrm{INK} 4 \mathrm{~b}}, \mathrm{p} 27^{\mathrm{KIP} 1}$ and $\alpha$-tubulin were purchased from Santa Cruz Biotechnology (Santa Cruz, CA). Antibodies for ERK1/2, phospho-ERK1/2 and the apoptosis sampler kit, which contains antibodies for the cleaved/ non-cleaved form of caspase-3/7/9 and PARP, were from Cell Signaling Technology (Danvers, MA). HRP-labeled secondary antibodies and the ECL Western blot detection reagent were purchased from GE Healthcare Bio-Science (Piscataway, NJ). Recombinant kinases of MEK1 (inactive), MEK2 (inactive), ERK2 (inactive), c-Raf (truncated form, active) and B-Raf (V599E, active) were obtained from Millipore (Billerica, MA).

Protein kinase assays. A Raf-MEK-ERK cascade kinase assay was carried out as previously described (21). Briefly, nonphosphorylated myelin basic protein, (MBP) (Millipore) was coated onto an ELISA plate, and the active form of B-Raf/c-Raf was mixed with unphosphorylated MEK1/MEK2 and ERK2 in $10 \mu \mathrm{M}$ ATP and $12.5 \mathrm{mM} \mathrm{MgCl}_{2}$ containing MOPS buffer in the presence of various concentrations of JTP-74057. The phosphorylation of MBP was detected by the anti-phosphoMBP antibody. Kinase inhibitory activities against a total of 99 kinases were tested by Millipore's kinase profiler at $10 \mu \mathrm{M}$ ATP.

Cell cultures and growth inhibition assays. Human colorectal cancer cell lines, HT-29, HCT-15, HCT116, COLO205, LS-174T,
SW480, SW620 and T84, were obtained from American Type Culture Collection (Manassas, VA). LoVo and COLO320 DM were from Health Science Research Resources Bank (Osaka, Japan). These cells were maintained in media recommended by the providers. Exponentially growing cells were precultured in 96-well tissue culture plates for $24 \mathrm{~h}$ and then exposed to JTP-74057. Cell growth was determined by an in vitro toxicology assay kit, sulforhodamine B based (Sigma-Aldrich, St. Louis, MO). For combination studies, two compounds were simultaneously added to the HT-29 cells and incubated for $72 \mathrm{~h}$. In the presence of various concentrations of compound $\mathrm{A}$, the $50 \%$ inhibitory concentration $\left(\mathrm{IC}_{50}\right)$ values of compound $\mathrm{B}$ were determined. Then, the fixed concentration of compound $\mathrm{A}$ versus the $\mathrm{IC}_{50}$ value of compound $\mathrm{B}$ was plotted. Conversely, the $\mathrm{IC}_{50}$ values of compound $\mathrm{A}$ were determined in the presence of various concentrations of compound $\mathrm{B}$ and plotted.

Western blot analysis. Western blot analysis of the cell lysates was carried out according to a standard method. Peroxidasebased chemiluminescence was developed by addition of ECL, and the bands were detected using an LAS 3000 image analyzer (Fuji Film, Tokyo, Japan).

Apoptosis assay. Cells were treated with various concentrations of JTP-74057 in 100-mm dishes for 3 or 4 days. Both floating and adherent cells were collected and fixed with $70 \%$ ethanol. After washing with PBS, the cells were suspended in $100 \mu \mathrm{l} / \mathrm{ml}$ RNase and $25 \mu \mathrm{l} / \mathrm{ml}$ propidium iodide (PI) and incubated at $37^{\circ} \mathrm{C}$ for $30 \mathrm{~min}$ in the dark. The DNA content of each single cell was determined using the flow cytometer Cytomics FC500 (Beckman Coulter, Brea, CA) or Guava EasyCyte plus (Millipore).

Nude mouse xenograft model. Female BALB/c-nu/nu mice obtained from Charles River Japan (Astugi, Japan) were used. On day 0, HT-29 cells or COLO205 cells suspended in ice-cold HBSS (-) were inoculated subcutaneously into the right flank of the mice at $5 \times 10^{6}$ cells $/ 100 \mu \mathrm{l} / \mathrm{site}$ or $1 \times 10^{6}$ cells $/ 100 \mu \mathrm{l} / \mathrm{site}$, respectively. The acetic acid-solvated form of JTP-74057 was dissolved in 10\% Cremophor EL-10\% PEG400 and was administered orally once daily for 14 days from the day when the mean tumor volume reached $100 \mathrm{~mm}^{3}$. The tumor length [L (mm)] and width [W $(\mathrm{mm})]$ were measured using a microgauge twice a week after commencement of dosing, and the tumor volume was calculated using the following formula: tumor volume $\left(\mathrm{mm}^{3}\right)$ $=\mathrm{L} \times \mathrm{W} \times \mathrm{W} / 2$. All procedures relating to the use of animals in this study were reviewed and approved by the Institutional Animal Care and Use Committee of Japan Tobacco.

Statistical analysis. The data were expressed as means \pm SD from the indicated number of samples. Multiple comparisons were assessed by one-way ANOVA followed by Dunnett's test (StatLight, Yukms, Tokyo, Japan) and two-sample comparisons were assessed by the Student's t-test. Differences were considered as significant at $\mathrm{p}<0.05$.

\section{Results}

JTP-74057 is a potent and highly specific MEK1/2 inhibitor. The inhibitory activity of JTP-74057 against MEK1/2 was tested using the Raf-MEK-ERK cascade kinase assay using 


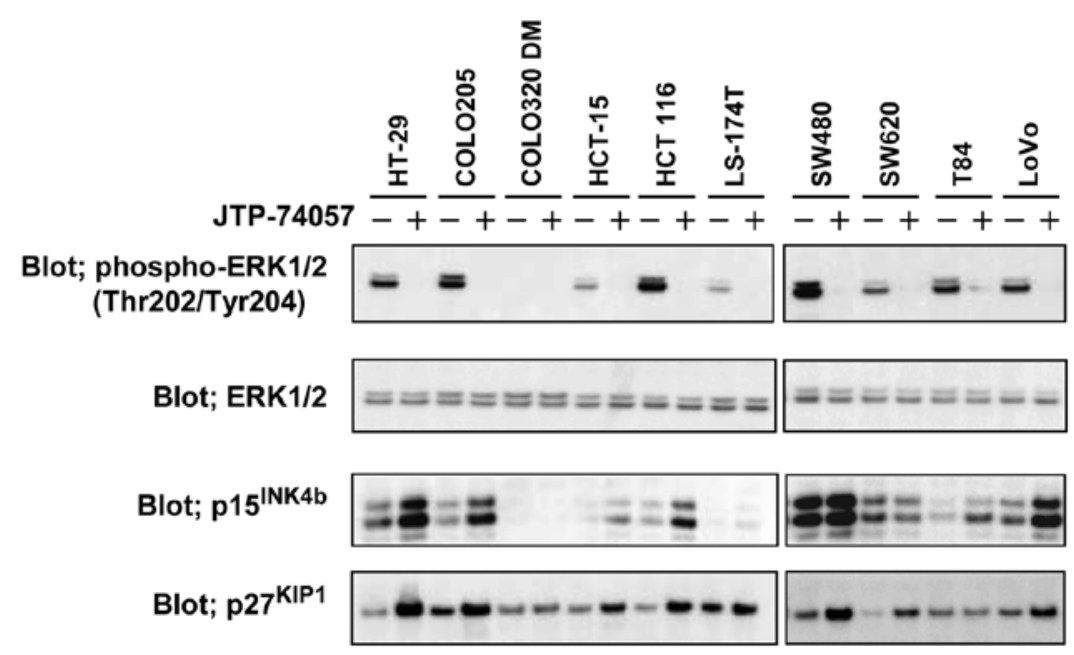

Figure 1. Effect of JTP-74057 on the phosphorylation of ERK1/2 and expression of p15 $5^{\mathrm{INK} 4 \mathrm{~b}}$ and $\mathrm{p} 27^{\mathrm{KIP1}}$ in various colorectal cancer cell lines. Ten colorectal cancer cell lines were treated with or without JTP-74057 at $100 \mathrm{nM}$ (in the case of HT-29, $10 \mathrm{nM}$ ). After 2 (ERK1/2) or $24 \mathrm{~h}$ (p15 $5^{\mathrm{INK} 4 \mathrm{~b}}$, p2 $7^{\mathrm{KIPl}}$ ), cells were lysed

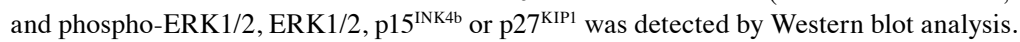

recombinant proteins. JTP-74057 inhibited the phosphorylation of MBP regardless of the isotype of Raf and MEK, with $\mathrm{IC}_{50}$ values ranging from 0.92 to $3.4 \mathrm{nM}$ (Table IA), which indicates JTP-74057 has 3- to 30-fold stronger activity than that of its parent compound JTP-70902 (21). JTP-74057 demonstrated no inhibition of the kinase activities of c-Raf, B-Raf, ERK1 and ERK2 (Table IB and data not shown), suggesting that JTP-74057 inhibits the Raf-MEK-ERK cascade reaction by inhibiting MEK1/2 activity. In addition, JTP-74057 did not show drastic inhibitory activity against the other 98 kinases examined (Table IB). The data are consistent with the finding that JTP-74057 directly binds to MEK1/2 by compound-immobilized affinity chromatography (data not shown). Together, these results indicate that JTP-74057 is a highly specific MEK1/2 inhibitor.

Anti-proliferative effect of JTP-74057 on 10 human colorectal cancer cell lines. Next, we examined the anti-proliferative effects of JTP-74057 using 10 human colorectal cancer cell lines. JTP-74057 inhibited the growth of 9 out of 10 cell lines
(Table II). HT-29 and COLO205 cells, which are known to have a constitutively active B-Raf mutant, were most sensitive to JTP-74057, showing subnanomolar $\mathrm{IC}_{50}$ values. The cell lines bearing a K-Ras mutation showed a wide range of sensitivity to JTP-74057. In contrast, COLO320 DM cells, bearing the wild-type gene in both B-Raf and K-Ras, were found to be resistant to JTP-74057 even at 10,000 nM. Similar results were reported regarding the other MEK inhibitors, PD0325901 and AZD6244 (15,22). All sensitive cell lines showed cell-cycle arrest at the $G_{1}$ phase after treatment with JTP-74057 for $24 \mathrm{~h}$ (data not shown). As we reported previously, MEK inhibitors induced the expression of CDK inhibitors $15^{\mathrm{INK} 4 \mathrm{~b}}$ and $\mathrm{p} 27^{\mathrm{KIP} 1}$, and we examined the expression level of p15 ${ }^{\mathrm{INK} 4 \mathrm{~b}}$ and $\mathrm{p} 27^{\mathrm{KIP} 1}$ in these cell lines after treatment with JTP-74057. As shown in Fig. 1, both $\mathrm{p} 15^{\mathrm{INK} 4 \mathrm{~b}}$ and $\mathrm{p} 27^{\mathrm{KIP} 1}$ were upregulated in the HT-29, COLO205, HCT-15, HCT116 and LoVo cells, whereas either $\mathrm{p} 15^{\mathrm{INK} 4 \mathrm{~b}}$ or $\mathrm{p} 27^{\mathrm{KIP} 1}$ was upregulated in the LS-174T, SW480, SW620 and T84 cells. Most importantly, the resistant cell line, COLO320 DM, showed no induction of $\mathrm{p} 15^{\mathrm{INK} 4 \mathrm{~b}}$ or $\mathrm{p} 27^{\mathrm{KIP} 1}$ by JTP-74057, suggesting that upregulation of either p15 $5^{\mathrm{INK} 4 \mathrm{~b}}$ or

Table I. MEK inhibitory activity and specificity of JTP-74057.

A. Inhibition of MEK enzyme activity by JTP-74057.

\begin{tabular}{llr}
\hline MEK & Activator & IC $_{50}$ value of JTP-74057 (nM) \\
\hline MEK1 & B-Raf & 3.4 \\
& c-Raf & 1.8 \\
MEK2 & B-Raf & 1.6 \\
& c-Raf & 0.92
\end{tabular}

${ }^{\mathrm{a}}$ Mean of 2 or 3 experiments. Inactive forms of MEK1 or MEK2 and ERK2 were added into myelin basic protein (MBP)-coated 96-well plates in the presence or absence of various concentrations of JTP-74057. A cascade kinase reaction was started by addition of active B-Raf or c-Raf with $10 \mu \mathrm{M}$ ATP and $12.5 \mathrm{mM} \mathrm{MgCl}_{2}$. After incubation at $30^{\circ} \mathrm{C}$ for $30 \mathrm{~min}$, the phosphorylation of MBP by ERK2 was detected with a peroxidase-labeled anti-phosphorylated MBP antibody. 
Table I. Continued.

B. Effect of JTP-74057 on various kinase activities (JTP-74057, $10 \mu \mathrm{M})$.

\begin{tabular}{|c|c|c|c|c|c|}
\hline Kinase & $\%$ of control & Kinase & $\%$ of control & Kinase & $\%$ of control \\
\hline Abl & 95 & Fyn & 101 & $\mathrm{PKC} \alpha$ & 101 \\
\hline Abl (T315I) & 112 & GSK $3 \alpha$ & 99 & PKC $\beta I$ & 108 \\
\hline ALK & 166 & GSK3 $\beta$ & 98 & РКС $\beta I I$ & 102 \\
\hline Arg & 111 & IGF-1R & 95 & $\mathrm{PKC} \gamma$ & 107 \\
\hline Aurora-A & 124 & $\mathrm{IKK} \alpha$ & 123 & $\mathrm{PKC} \delta$ & 97 \\
\hline Axl & 114 & $\mathrm{IKK} \beta$ & 111 & $\mathrm{PKC} \varepsilon$ & 103 \\
\hline $\mathrm{Bmx}$ & 93 & IR & 129 & $\mathrm{PKC \eta}$ & 107 \\
\hline BTK & 104 & $\mathrm{JNK} 1 \alpha 1$ & 104 & $\mathrm{PKCl}$ & 83 \\
\hline CaMKIV & 97 & JNK $2 \alpha 2$ & 97 & $\mathrm{PKC} \mu$ & 111 \\
\hline CDK1/cyclin B & 104 & JNK3 & 76 & $\mathrm{PKC} \theta$ & 114 \\
\hline CDK2/cyclin A & 101 & Lck & 110 & PKC $\zeta$ & 102 \\
\hline CDK2/cyclin E & 104 & Lyn & 95 & PKD2 & 98 \\
\hline CDK3/cyclin E & 101 & MAPK1 (ERK1) & 86 & Plk3 & 91 \\
\hline CDK5/p35 & 102 & MAPK2 (ERK2) & 108 & PRAK & 91 \\
\hline CDK6/cyclin D3 & 107 & MAPKAP-K2 & 109 & PRK2 & 98 \\
\hline CDK7/cyclin H/MAT1 & 106 & MEK1 & 28 & Ret & 109 \\
\hline CHK1 & 113 & Met & 150 & ROCK-II & 99 \\
\hline CHK2 & 98 & MKK6 & 70 & Ros & 107 \\
\hline CK1ठ & 105 & MKK7 $\beta$ & 109 & Rse & 112 \\
\hline CK2 & 101 & MSK1 & 111 & Rsk1 & 116 \\
\hline c-Raf & 108 & MST2 & 94 & Rsk2 & 94 \\
\hline CSK & 97 & NEK2 & 119 & Rsk3 & 102 \\
\hline $\mathrm{cSRC}$ & 82 & p70S6K & 104 & SAPK2a & 84 \\
\hline EGFR & 104 & PAK2 & 96 & SAPK2b & 104 \\
\hline EphA2 & 93 & PAR- $1 B \alpha$ & 116 & SAPK3 & 97 \\
\hline EphB2 & 107 & $\operatorname{PDGFR} \alpha$ & 111 & SAPK4 & 109 \\
\hline EphB4 & 124 & $\operatorname{PDGFR} \beta$ & 111 & SGK & 114 \\
\hline ErbB4 & 101 & PDK1 & 107 & Syk & 74 \\
\hline Fes & 95 & Pim-1 & 94 & Tie2 & 103 \\
\hline FGFR3 & 98 & PKA & 98 & TrkA & 59 \\
\hline $\mathrm{Fgr}$ & 86 & $\mathrm{PKB} \alpha$ & 107 & TrkB & 120 \\
\hline Flt3 & 107 & $\mathrm{PKB} \beta$ & 103 & Yes & 104 \\
\hline Fms & 128 & PKB $\gamma$ & 109 & ZAP-70 & 111 \\
\hline
\end{tabular}

Abl, Abl tyrosine kinase; T315I, T315I, mutations of Thr-315 to Ile; ALK, anaplastic lymphoma kinase; Arg, Abl-related gene product tyrosine kinase; Aurora-A, Aurora-A kinase; Axl, Axl receptor tyrosine kinase; Bmx, BMX non-receptor tyrosine kinase; BTK, bruton agammaglobulinemia tyrosine kinase; CaMK, calcium/calmodulin-dependent protein kinase; CDK, cyclin-dependent kinase; MAT, menage a trois; CHK, checkpoint kinase; CK, casein kinase; c-RAF, Raf proto-oncogene serine/threonine protein kinase; CSK, C-terminal Src kinase; cSRC, SRC proto-oncogene tyrosine protein kinase; EGFR, epidermal growth factor receptor kinase; EphA2, Ephrin-A2 receptor tyrosine protein kinase; EphB2, Ephrin-B2 receptor tyrosine protein kinase; EphB4, ephrin-B4 receptor tyrosine protein kinase; ErbB4, ErbB4 tyrosine kinase; Fes, Fes tyrosine protein kinase; FGFR, fibroblast growth factor receptor kinase; Fgr, Fgr tyrosine protein kinase; Flt, FMS-like tyrosine protein kinase; Fms, Fms tyrosine protein kinase; Fyn, Fyn tyrosine protein kinase; GSK, glycogen synthase kinase; IGF-1R, insulin-like growth factor-1 receptor kinase; IKK, IкB kinase; IR, insulin receptor; JNK, c-Jun N-terminal kinase; Lck, lymphocyte-specific protein-tyrosine kinase; Lyn, Lyn protein tyrosine kinase; MAPK, mitogen-activated protein kinase; ERK, extracellular signal-regulated kinase; MAPKAP-K2, MAPK-activated protein kinase-2; MEK, MAPK/ERK kinase; Met, c-Met receptor tyrosine kinase; MKK, MAPK kinase; MSK, mitogen- and stress-activated protein kinase; MST, mammalian homologue STE20-like kinase; NEK, non-inherited maternal MHC antigens (NIMA)-related kinase; p70S6K, p70S6 kinase; PAK, p21-activated protein kinase; PAR-1B $\alpha$, protease-activated receptor 1B $\alpha$; PDGFR, platelet-derived growth factor receptor; PDK1, 3'-phosphoinositide-dependent kinase-1; Pim-1, Pim-1 protein kinase; PKA, cAMP-dependent protein kinase; PKB, protein kinase B; PKC, protein kinase C; PKD, protein kinase D; Plk3, polo-like kinase-3; PRAK, p38-related/activated protein kinase; PRK, protein kinase C-related kinase; Ret, ret receptor tyrosine kinase; ROCK-II, Rho-associated coiled-coil-containing protein kinase 2; Ros, Ros receptor protein tyrosine kinase; Rse, Rse receptor-type tyrosine kinase; Rsk, p90 ribosomal S6 kinase; SAPK, stress-activated protein kinase; SGK, serum/glucocorticoid-inducible kinase; Syk, spleen tyrosine kinase; Tie2, Tie-2 receptor tyrosine kinase; Trk, tyrosine kinase; Yes, Yes kinase; ZAP-70, $\zeta$-chain (TCR)-associated protein kinase $70 \mathrm{kDa}$. 
A

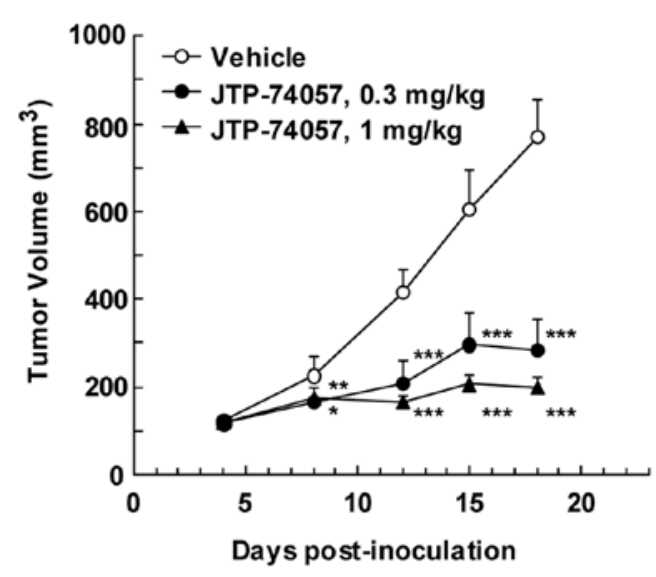

B

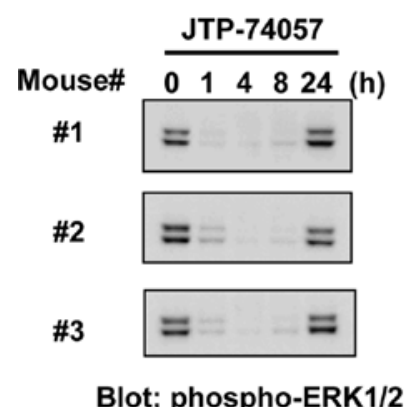

Blot; phospho-ERK1/2

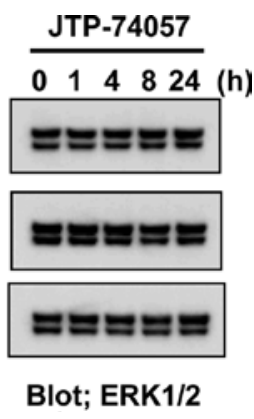

C

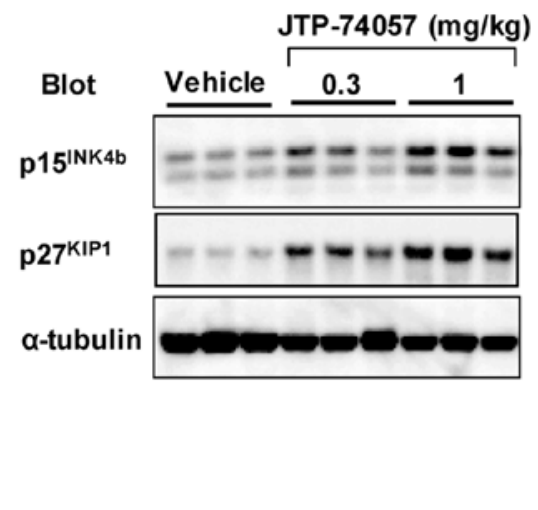

D

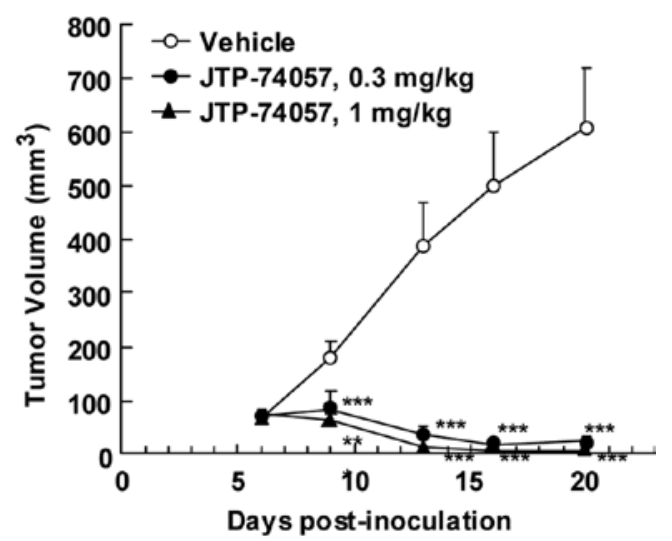

Figure 2. Antitumor activity of JTP-74057 in a colorectal cancer cell-xenografted nude mouse model. HT-29 (A) or COLO205 (D) cells were inoculated subcutaneously into the right flank of nude mice, and JTP-74057 or vehicle was administered orally once daily for 14 days starting from 5 days (HT-29) or 7 days (COLO205) after inoculation (open circle, vehicle; closed circle, $0.3 \mathrm{mg} / \mathrm{kg}$; closed triangle, $1 \mathrm{mg} / \mathrm{kg}$ ). Tumor volume was measured twice a week. The results are expressed as mean $\pm \mathrm{SD}(\mathrm{n}=6) .{ }^{*} \mathrm{p}<0.05,{ }^{* *} \mathrm{p}<0.01,{ }^{* * * *} \mathrm{p}<0.001$ versus vehicle (Dunnett's test). The phosphorylation of ERK1/2 (B) and the expression of p15 ${ }^{\mathrm{INK} 4 \mathrm{~b}}$ and $\mathrm{p} 27^{\mathrm{KIPl}}$ (C) in HT-29 xenograft tumors after treatment with JTP-74057. (B) Single oral dose of $1 \mathrm{mg} / \mathrm{kg}$ JTP-74057 was administered to nude mice bearing the HT-29 xenografts $(n=3)$. The tumor tissues were excised at indicated time points and homogenized in lysis buffer. The resultant homogenate was centrifuged and the supernatant was used for Western blot analysis. (C) Nude mice bearing the HT-29 xenografts were treated with vehicle or JTP-74057 once daily for 14 days, and the tumors were excised and homogenized. The expression level of p15 $5^{\mathrm{INK} 4 \mathrm{~b}}, \mathrm{p} 27^{\mathrm{KIPI}}$ and $\alpha$-tubulin was analyzed by Western blot analysis.

Table II. In vitro growth inhibitory activities of JTP-74057 against human colorectal cancer cell lines.

\begin{tabular}{lcc}
\hline Cell line & Ras/B-Raf mutation & $\mathrm{IC}_{50}$ value $(\mathrm{nM})^{\mathrm{a}}$ \\
\hline HT-29 & B-Raf & 0.48 \\
COLO205 & B-Raf & 0.52 \\
COLO320 DM & WT & $>10,000$ \\
HCT-15 & K-Ras & 174 \\
HCT 116 & K-Ras & 5.7 \\
LS-174T & K-Ras & 4.1 \\
SW480 & K-Ras & 8.5 \\
SW620 & K-Ras & 2.3 \\
T84 & K-Ras & 36 \\
LoVo & K-Ras & 2.2 \\
\hline
\end{tabular}

${ }^{\mathrm{a}}$ Mean of 3 experiments. Various human colorectal cancer cell lines were treated with various concentrations of JTP-74057 for $72 \mathrm{~h}$. Viable cell number was determined by Sulforhodamine B assay. $\mathrm{p} 27^{\mathrm{KIP} 1}$ is a prerequisite for growth inhibition of MEK inhibitors.

We also tested the phophorylation level of ERK1/2 by Western blotting. Constitutive ERK phosphorylation was detected in all the cell lines except for COLO320 DM, and it was inhibited by JTP-74057 (Fig. 1). These results suggest that growth inhibitory activity of JTP-74057 is strongly dependent on the MEK-ERK pathway in tumor cells.

Oral dosing of JTP-74057 resulted in tumor growth inhibition in a nude mouse xenograft model. JTP-74057 was evaluated in mice for antitumor activity against HT-29 xenografts. HT-29 cells were inoculated into nude mice, and JTP-74057 was orally administered once daily for 14 days. Both $0.3 \mathrm{mg} /$ $\mathrm{kg}$ and $1 \mathrm{mg} / \mathrm{kg}$ of JTP-74057 were effective in inhibiting the HT-29 xenograft growth, and $1 \mathrm{mg} / \mathrm{kg}$ of JTP-74057 blocked the tumor increase almost completely (Fig. 2A). Neither significant changes in body weight nor gross lesions at autopsy were observed in the mice treated with $1 \mathrm{mg} / \mathrm{kg}$ of JTP-74057. We 
A

HT-29
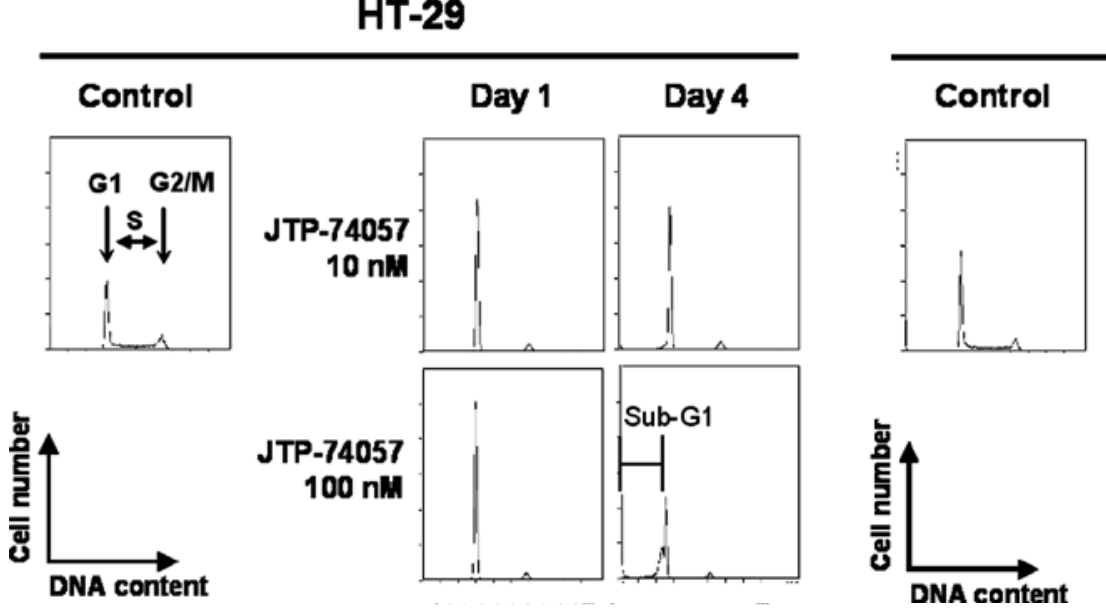

COLO205

B
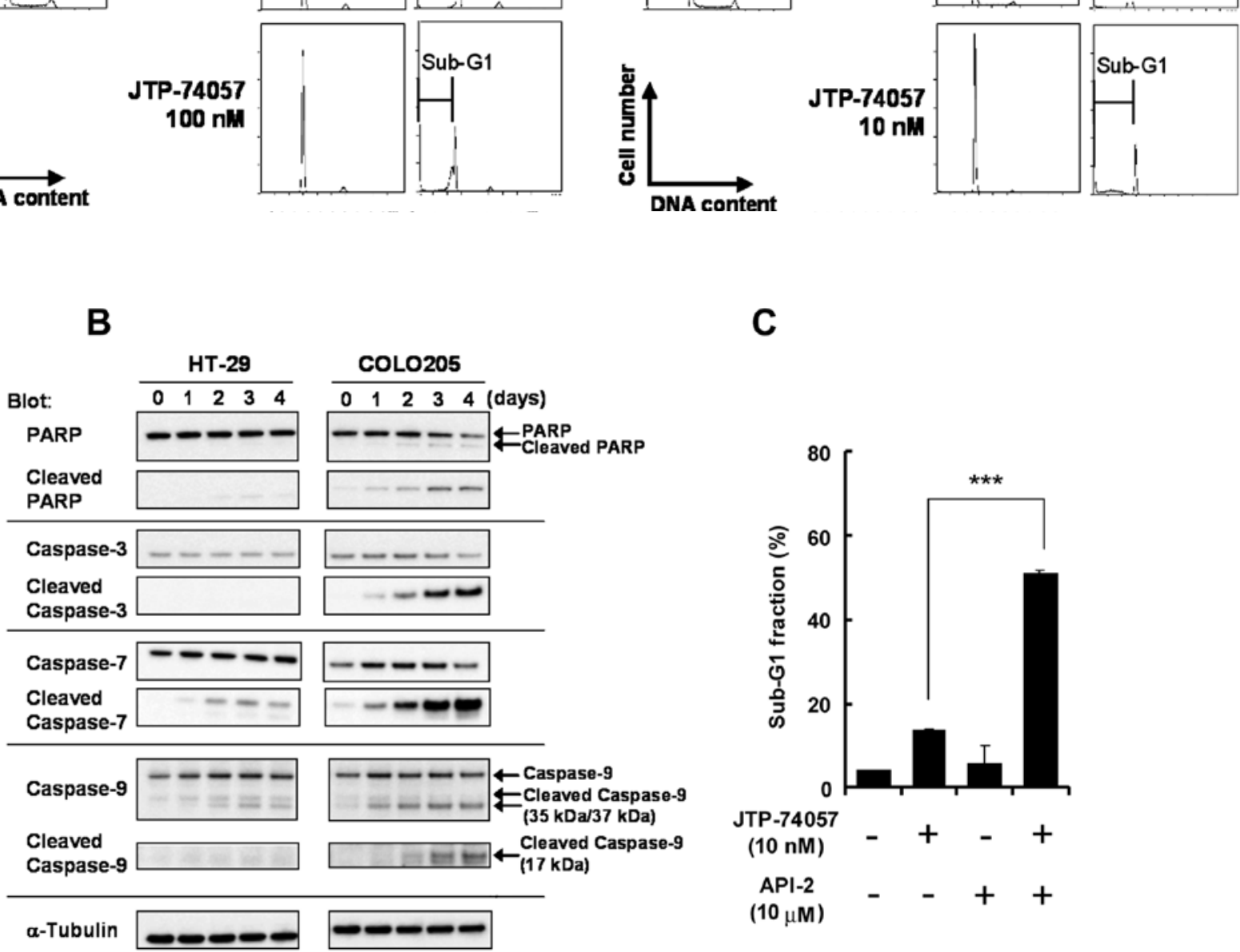

C

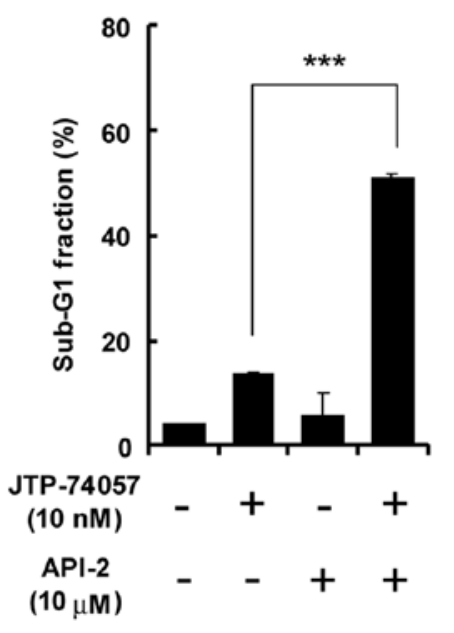

Figure 3. Differential sensitivity of apoptosis against JTP-74057. (A) HT-29 and COLO205 cells were treated with JTP-74057 at the indicated concentrations. On day 1 or day 4 , the cells were collected and fixed by $70 \%$ ethanol. Intracellular DNA in the fixed cells was stained with propidium iodide (PI). DNA content was analyzed by a flow cytometer. Untreated cells on day 0 were analyzed as a control. The gate in the graph $(H)$ indicates sub- $\mathrm{G}_{1}$ fraction cells. $(B)$ Cells treated with $10 \mathrm{nM}$ of JTP-74057 were collected daily from day 1 to day 4. Recovered cells were lysed and analyzed by Western blotting with indicated

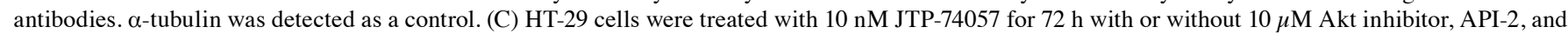
analyzed for sub- $\mathrm{G}_{1}$ fraction. ${ }^{* * *} \mathrm{p}<0.001$ versus JTP-74057 alone (Student's t-test).

also found that the phosphorylation of ERK1/2 was completely inhibited in the established tumor tissues by single oral dose of $1 \mathrm{mg} / \mathrm{kg} \mathrm{JTP-74057} \mathrm{(Fig.} \mathrm{2B),} \mathrm{and} \mathrm{both} \mathrm{p15}{ }^{\mathrm{INK} 4 \mathrm{~b}}$ and p27 $7^{\mathrm{KIP} 1}$ protein levels were upregulated after 14 days of treatment with JTP-74057 (Fig. 2C). These results indicate that JTP-74057 exerts an antitumor effect in vivo by the mechanisms of action that were demonstrated in the in vitro studies. The antitumor activity of JTP-74057 was also evaluated in the COLO205 xenograft model. As shown in Fig. 2D, tumor regression was observed even at a dose of $0.3 \mathrm{mg} / \mathrm{kg}$. At a dose of $1 \mathrm{mg} / \mathrm{kg}$, a complete regression was obtained in 4 out of 6 mice in which the tumor degenerated to the point that tumor volume was not measurable. Again, neither significant changes in body weight nor gross lesions at autopsy were observed in the mice treated with JTP-74057.

Apoptosis induction by JTP-74057 in HT-29 and COLO205 cells in vitro. Despite the fact that JTP-74057 inhibited the proliferation of HT-29 and COLO205 cells with comparable $\mathrm{IC}_{50}$ values in vitro, COLO205 tumors were much more sensitive to JTP-74057 than HT-29 tumors in vivo. To address this issue, apoptosis induction by JTP-74057 was analyzed in vitro. Both cell lines were treated with JTP-74057 for 4 days. On days 1 and 4, cells were collected and the intracellular DNA was measured by flow cytometry. As shown in Fig. 3A, on day 1 , the $G_{1}$ peak was elevated compared to that on day 0 at all 
A

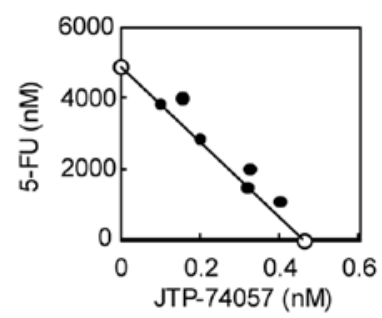

B

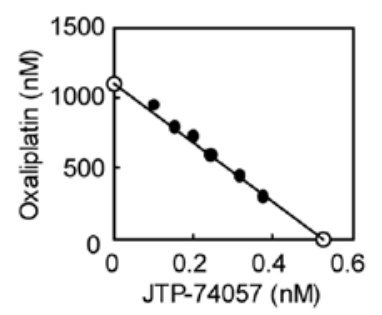

C

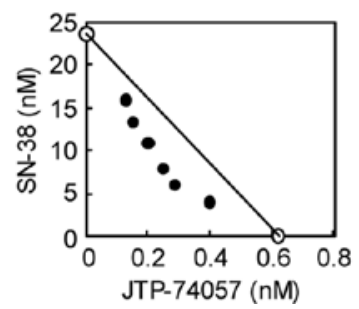

D

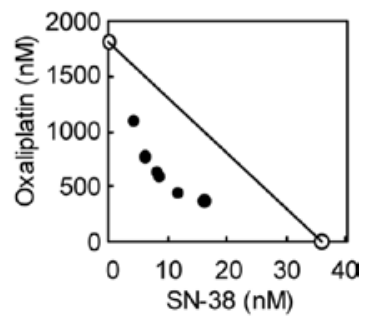

E

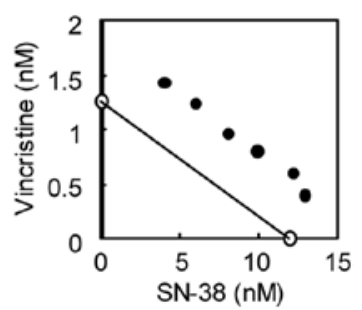

Figure 4. Combination effect of JTP-74057 with clinically used chemotherapeutic agents. Two compounds were simultaneously added to HT-29 cells Seventy-two hours after treatment, the viable cell number was analyzed by Sulforhodamine B assay. In the presence of various concentrations of the first compound, the $\mathrm{IC}_{50}$ values of the second compound were determined. The concentrations of the first compound and the $\mathrm{IC}_{50}$ values of the second compound were plotted. The line connecting the $\mathrm{IC}_{50}$ values in monotreatment (y-intercept and $\mathrm{x}$-intercept) was compared with the $\mathrm{IC}_{50}$ plots in the combination treatment to evaluate the effect as follows: additive effect, the plot in combination treatment is on the line; synergistic effect, the plot in combination treatment is below the line; antagonism, the plot in combination treatment is above the line. (A) JTP-74057 with 5-FU; (B) JTP-74057 with oxaliplatin; (C) JTP-74057 with SN-38; (D) SN-38 with oxaliplatin; (E) SN-38 with vincristine.

concentrations of JTP-74057 in both cell lines, indicating the cell cycles were arrested in $G_{1}$. Thereafter, the $G_{1}$ peak decreased in the HT-29 cells exposed to $100 \mathrm{nM}$ JTP-74057 and in COLO205

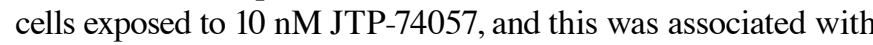
the appearance of the sub- $\mathrm{G}_{1}$ fraction to the left of the peak of $\mathrm{G}_{1}$. These results suggest that JTP-74057 induces apoptosis both in HT-29 and COLO205 cells, but that COLO205 cells are more sensitive to JTP-74057 than HT-29 cells in terms of apoptosis induction. Concomitant with the appearance of the sub- $\mathrm{G}_{1}$ fraction, treatment with $10 \mathrm{nM}$ of JTP-74057 gradually activated caspases only in COLO205 cells, which was confirmed by Western blotting against the cleaved forms of caspase-3, -7, -9 and PARP (Fig. 3B). The cleavage of caspase-3, -7, -9 and PARP was observed only at $100 \mathrm{nM}$ of JTP-74057 in HT-29 cells (data not shown). Since it has been recently reported that the PI3K-Akt pathway suppresses MEK inhibitor-induced apoptosis (23-25), we then performed the

combination treatment of $10 \mathrm{nM}$ of JTP-74057, which did not induce apoptosis, and Akt inhibitor, API-2, in HT-29 cells. As shown in Fig. 3C, API-2 significantly enhanced apoptosis in HT-29 cells treated with JTP-74057 as expected.

Combination effect of JTP-74057 and standard-of-care agents on the growth inhibition in HT-29 cells. Finally, the combination effects of JTP-74057 with standard-of-care agents for colorectal cancer, 5-FU, oxaliplatin and SN-38 (the active metabolite of irinotecan) were investigated in vitro using the isobologram method as described in Materials and methods. JTP-74057 showed an additive effect in combination with 5-FU or oxaliplatin, and a synergistic effect in combination with $\mathrm{SN}-38$ (Fig. $4 \mathrm{~A}-\mathrm{C}$ ). The $\mathrm{IC}_{50}$ values of $\mathrm{SN}-38$ added with oxaliplatin were plotted below the line connecting the $\mathrm{IC}_{50}$ values in mono-treatment, showing synergism (Fig. 4D). The data are consistent with a previous report (26). Likewise, the effect of SN-38 treatment in combination with vincristine demonstrated antagonism in terms of the isobologram (Fig. 4E), consistent with a previous report (27).

\section{Discussion}

The MEK-ERK pathway regulates the expression of various cell cycle-related proteins. MEK inihibitors induce CDK inhibitors (CKIs), p15 ${ }^{\mathrm{INK} 4 \mathrm{~b}}$ and p27 ${ }^{\mathrm{KIP} 1}$, and downregulate cyclin $\mathrm{D} 1$, cyclin $\mathrm{E}$ and cyclin $\mathrm{A}$, which in turn leads to reactivation of retinoblastoma gene $(\mathrm{RB})$ protein and repression of a number of cell cycle-promoting molecules, so that finally the cell cycle is arrested at the $G_{1}$ phase $(15,21,28-30)$. In the present study, we utilized a panel of 10 human colorectal cancer cell lines and investigated the relationship between CKI induction and growth inhibition by the MEK inhibitor, JTP-74057. JTP-74057 inhibited cell proliferation of all the cell lines except for COLO320 DM cells, and either p15 ${ }^{\mathrm{INK} 4 \mathrm{~b}}$ or p $27^{\mathrm{KIP} 1}$, or both, were upregulated after the treatment in these sensitive cell lines. On the other hand, neither $\mathrm{p} 15^{\mathrm{INK} 4 \mathrm{~b}}$ nor p27 ${ }^{\mathrm{KIP} 1}$ was induced by JTP-74057 in COLO320 DM cells. In contrast, we previously demonstrated that $\mathrm{p} 15^{\mathrm{INK} 4 \mathrm{~b}}(-/-)$ mouse embryonic fibroblasts (MEFs) were more resistant to the growth-inhibitory effect of the MEK inhibitor JTP-70902 than wild-type MEFs (21). Taken together, these results suggest that $\mathrm{p} 15^{\mathrm{INK} 4 \mathrm{~b}}$ and $\mathrm{p} 27^{\mathrm{KIP} 1}$ at least partially play roles in cell cycle arrest by a MEK inhibitor.

However, the relative importance of these events in growth arrest may vary in each cell line. For instance, there are two conflicting reports regarding the necessity of $\mathrm{p} 27^{\mathrm{KIP} 1}$ induction in the cell cycle arrest by MEK inhibitor U0126. It was indispensable in pancreatic cell lines (29), but not in colorectal cancer cell lines (30). We therefore hypothesized that other factors such as induction of apoptosis may be involved in the growth suppression by MEK inhibitors.

We next performed an in vivo study and found that JTP-74057 exerted excellent efficacy both in HT-29 and COLO205 xenograft models. As shown in Fig. 2B, COLO205 tumors were almost completely diminished by daily oral administration with JTP-74057 at a dose of $1 \mathrm{mg} / \mathrm{kg} / \mathrm{day}$. This efficacy was superior to those of the second generation MEK inhibitors, as PD0325901 or AZD6244 induced the tumor regression at 25 or $50 \mathrm{mg} / \mathrm{kg} / \mathrm{day}$ in the COLO 205 
xenograft model, respectively $(15,22)$. Tumor regression by JTP-74057 was observed in the COLO205 tumors, but not in the HT-29 tumors, despite comparable $\mathrm{IC}_{50}$ values observed in the in vitro assay. As there was no substantial difference in the engraftment rates or the doubling time between the two cell lines, it is not likely that host defense against these cell lines is different. By further analysis in vitro, we found that the COLO205 cells were much more sensitive to JTP-74057induced apoptosis than the HT-29 cells. This may be one of the major reasons why JTP-74057 achieved tumor regression only in the COLO205 xenograft model.

Many proteins involved in apoptosis are regulated by the MEK-ERK pathway (2). Indeed, MEK inhibition by JTP-74057 resulted in caspase-dependent apoptosis. Many types of cancer cells acquire resistence to apoptosis by activating several signal transduction pathways such as the nuclear factor (NF)- $\mathrm{KB}$ pathway, the MEK-ERK pathway or the PI3K-Akt pathway. Mutation of PIK3CA or PTEN is frequently observed in colorectal cancers $(31,32)$. It has been reported that sensitivity to the anti-proliferative effect of AZD6244 was rescued in the resistant cell lines CO115 and DLD-1 when the cells were treated with PI3K inhibitor LY294002 (28). HT-29 cells, but not COLO205 cells, were reported to have a mutation in PIK3CA, suggesting that mutation status in the PI3K pathway may be a possible factor in acquiring resistance to apoptosis. In addition, a recent study demonstrated that GSK1120212 (JTP-74057 DMSO solvate) tends to show cytostatic effects rather than cytotoxic effects in cell lines harboring PI3K or PTEN mutations compared to those of PI3K/PTEN wildtype cells (33). These reports are consistent with the results shown in Fig. 3C, which indicate that the Akt inhibitor API-2 significantly enhanced apoptosis in HT-29 cells treated with JTP-74057. Importantly, GSK1120212 (JTP-74057 DMSO solvate) is being evaluated in a phase I clinical trial in combination with the orally available Akt inhibitor GSK2141795. Cell proliferation assays have been utilized as a gold standard assay to predict the sensitivity of tumor cells to antitumor agents. However, this may not be sufficient to predict absolute efficacy in vivo. Our observations in this study raise a possibility that an apoptosis assay may also be valuable for prediction of in vivo efficacy with standard cell proliferation assay. Combination therapy is a common approach in cancer chemotherapy. For colorectal cancers, the standard-of-care drugs 5-FU, oxaliplatin and irinotecan are often combined (34). Those agents are known to be highly effective against actively growing cells. In contrast, JTP-74057 induces $\mathrm{G}_{1}$ cell cycle arrest in multiple malignant tumor cells. Thus, there was a concern that the efficacy of those standard-of-care drugs might be reduced when JTP-74057 was combined with them. However, JTP-74057 showed an additive effect in combination with 5-FU or oxaliplatin, and a synergistic effect in combination with SN-38. We also examined the combination effect of JTP-74057 with these agents following sequential exposure. JTP-74057 did not show an antagonistic effect in combination when the anticancer drugs were added $24 \mathrm{~h}$ after exposure to JTP-74057 or vice versa (data not shown). These results suggest that JTP-74057 may enhance the therapeutic benefit of clinically used anticancer drugs.

In conclusion, we identified a novel MEK1/2 inhibitor JTP-74057 (also called GSK1120212) from a high-throughput screening for compounds that induce expression of $\mathrm{p} 15^{\mathrm{INK} 4 \mathrm{~b}}$. JTP-74057 is selective and orally available. In addition, the antitumor activity of JTP-74057 appears to be superior to those of the second generation MEK inhibitors in preclinical animal models. Furthermore, sensitivity to JTP-74057-induced apoptosis may be an important factor for the estimation of in vivo efficacy, and the sensitivity was enhanced by an Akt inhibitor. GSK1120212 (JTP-74057 DMSO solvate) is now being evaluated in a phase III clinical trial against melanoma patients with B-Raf mutations (NCT01240562), and the results of a phase I study revealed that in 29 B-Raf mutant melanoma patients, 2 complete responses and 6 partial responses were observed (ESMO 2010: http://www.esmo.org/events/ milan-2010-congress/news/view.html?tx_ttnews\%5Btt_ news $\% 5 \mathrm{D}=978 \&$ tx_ttnews $\% 5 \mathrm{BbackPid} \% 5 \mathrm{D}=585 \& \mathrm{cHash}=$ 86cc01353e). Furthermore, GSK1120212 is also being evaluated in a phase I clinical trials in combination with the Akt inhibitor GSK2141795, the BRAF inhibitor GSK2118436, the mTOR inhibitor everolimus, the PI3K inhibitor GSK2126458 or the PI3K inhibitor BKM120 (ClinicalTrials.gov: http:// clinicaltrials.gov/ct2/results?term=GSK1120212). Taken together, JTP-74057 (GSK1120212), originally identified by screening for $\mathrm{p} 15^{\mathrm{INK} 4 \mathrm{~b}}$ upregulation, is a promising and novel MEK inhibitor for the treatment of patients suffering from a variety of cancers.

\section{Acknowledgements}

We thank Hiroshi Chatani, Tomoko Matsui, Junya Kakegawa, Takayuki Yoshida and Miki Wakada for technical support, Toyomichi Nanayama and Yoshihiro Watanabe for critical advice and discussions, and Hisashi Kawasaki and Hiroyuki Abe for providing the compounds.

\section{References}

1. Sebolt-Leopold JS and Herrera R: Targeting the mitogenactivated protein kinase cascade to treat cancer. Nat Rev Cancer 4: 937-947, 2004

2. McCubrey JA, Milella M, Tafuri A, et al: Targeting the Raf/ MEK/ERK pathway with small-molecule inhibitors. Curr Opin Investig Drugs 9: 614-630, 2008.

3. Bos JL: Ras oncogenes in human cancer: a review. Cancer Res 49: 4682-4689, 1989.

4. Adjei AA: Blocking oncogenic Ras signaling for cancer therapy. J Natl Cancer Inst 93: 1062-1074, 2001.

5. Davies H, Bignell GR, Cox C, et al: Mutations of the BRAF gene in human cancer. Nature 417: 949-954, 2002.

6. Thomas NE: BRAF somatic mutations in malignant melanoma and melanocytic naevi. Melanoma Res 16: 97-103, 2006.

7. Yuen ST, Davies H, Chan TL, et al: Similarity of the phenotypic patterns associated with BRAF and KRAS mutations in colorectal neoplasia. Cancer Res 62: 6451-6455, 2002.

8. Cohen Y, Xing M, Mambo E, et al: BRAF mutation in papillary thyroid carcinoma. J Natl Cancer Inst 95: 625-627, 2003.

9. Kimura ET, Nikiforova MN, Zhu Z, Knauf JA, Nikiforov YE and Fagin JA: High prevalence of BRAF mutations in thyroid cancer: genetic evidence for constitutive activation of the RET/PTC-RAS-BRAF signaling pathway in papillary thyroid carcinoma. Cancer Res 63: 1454-1457, 2003.

10. Blume-Jensen $P$ and Hunter T: Oncogenic kinase signaling. Nature 411: 355-365, 2001.

11. Porter AC and Vaillancourt RR: Tyrosine kinase receptoractivated signal transduction pathways which lead to oncogenesis. Oncogene 17: 1343-1352, 1998.

12. Sebolt-Leopold JS, Dudley DT, Herrera R, et al: Blockade of the MAP kinase pathway suppresses growth of colon tumors in vivo. Nat Med 5: 810-816, 1999. 
13. Rinehart J, Adjei AA, Lorusso PM, et al: Multicenter phase II study of the oral MEK inhibitor, CI-1040, in patients with advanced non-small-cell lung, breast, colon, and pancreatic cancer. J Clin Oncol 22: 4456-4462, 2004.

14. Yeh TC, Marsh V, Bernat BA, et al: Biological characterization of ARRY-142886 (AZD6244), a potent, highly selective mitogen-activated protein kinase kinase $1 / 2$ inhibitor. Clin Cancer Res 13: 1576-1583, 2007.

15. Solit DB, Garraway LA, Pratilas CA, et al: BRAF mutation predicts sensitivity to MEK inhibition. Nature 439: 358-362, 2006.

16. Sowa Y, Orita T, Minamikawa-Hiranabe S, Mizuno T, Nomura $\mathrm{H}$ and Sakai T: Histone deacetylase inhibitor activates the WAF1/ Cip1 gene promoter through the Sp1 sites. Biohem Biophys Res Commun 241: 142-150, 1997.

17. Koyama M, Matsuzaki Y, Yogosawa S, et al: ZD1839 induces p1 $5^{\mathrm{INK} 4 \mathrm{~b}}$ and causes $\mathrm{G}_{1}$ arrest by inhibiting the mitogen-activated protein kinase/extracellular signal-required kinase pathway. Mol Cancer Ther 6: 1579-1587, 2007.

18. Nishimura N, Furukawa Y, Sutheesophon K, et al: Suppression of ARG kinase activity by STI571 induces cell cycle arrest through up-regulation of CDK inhibitor p18/INK4c. Oncogene 22: 4074-4082, 2003.

19. Le XF, Pruefer F and Bast RC Jr: HER2-targeting antibodies modulate the cyclin-dependent kinase inhibitor $\mathrm{p} 27^{\mathrm{Kipl}} \mathrm{via}$ multiple signaling pathways. Cell Cycle 4: 87-95, 2005.

20. Shindoh N, Mori M, Terada Y, et al: YM753, a novel histone deacetylase inhibitor, exhibits antitumor activity with selective, sustained accumulation of acetylated histones in tumors in the WiDr xenograft model. Int J Oncol 32: 545-555, 2008.

21. Yamaguchi T, Yoshida T, Kurachi R, et al: Identification of JTP-70902, a p15 $5^{\mathrm{INK} 4 \mathrm{~b}}$-inductive compound, as a novel MEK1/2 inhibitor. Cancer Sci 98: 1809-1816, 2007.

22. Davies BR, Logie A, McKay JS, et al: AZD6244 (ARRY-142886), a potent inhibitor of mitogen-activated protein kinase/extracellular signal-regulated kinase kinase 1/2 kinases: mechanism of action in vivo, pharmacokinetic/pharmacodynamic relationship, and potential for combination in preclinical models. Mol Cancer Ther 6: 2209-2219, 2007.

23. Mirzoeva OK, Das D, Heiser LM, et al: Basal subtype and MAPK/ERK kinase (MEK)-phosphoinositide 3-kinase feedback signaling determine susceptibility of breast cancer cells to MEK inhibition. Cancer Res 69: 565-572, 2009.
24. Hoeflich KP, O'Brien C, Boyd Z, et al: In vivo antitumor activity of MEK and phosphatidylinositol 3-kinase inhibitors in basallike breast cancer models. Clin Cancer Res 15: 4649-4664, 2009.

25. Yang JY, Chang CJ, Xia W, et al: Activation of FOXO3a is sufficient to reverse mitogen-activated protein/extracellular signal-regulated kinase kinase inhibitor chemoresistance in human cancer. Cancer Res 70: 4709-4718, 2010.

26. Zeghari-Squalli N, Raymond E, Cvitkovic E and Goldwasser F: Cellular pharmacology of the combination of the DNA topoisomerase I inhibitor SN-38 and the diaminocyclohexane platinum derivative oxaliplatin. Clin Cancer Res 5: 1189-1196, 1999.

27. Akutsu M, Suzuki K, Tsunoda S, Kano Y and Miura Y: Efficacy of combination therapy of irinotecan (CPT-11) and other anticancer drugs: evaluation in B-lymphoid tumor cell lines. Jpn J Cancer Chemotherapy 21: 1607-1611, 1994.

28. Balmanno K, Chell SD, Gillings AS, Hayat S and Cook SJ: Intrinsic resistance to the MEK1/2 inhibitor AZD6244 (ARRY142886) is associated with weak ERK1/2 signalling and/or strong PI3K signalling in colorectal cancer cell lines. Int J Cancer 125: 2332-2341, 2009.

29. Gysin S, Lee SH, Dean NM and McMahon M: Pharmacologic inhibition of $\mathrm{RAF} \rightarrow \mathrm{MEK} \rightarrow \mathrm{ERK}$ signaling elicits pancreatic cancer cell cycle arrest through induced expression of $\mathrm{p} 27^{\mathrm{Kip} 1}$ Cancer Res 65: 4870-4880, 2005

30. Tetsu $\mathrm{O}$ and McCormick F: Proliferation of cancer cells despite CDK2 inhibition. Cancer Cell 3: 233-245, 2003.

31. Samuels Y, Wang Z and Bardelli A: High frequency of mutations of the PIK3CA gene in human cancers. Science 304: 554, 2004.

32. Nassif NT, Lobo GP, Wu X, et al: PTEN mutations are common in sporadic microsatellite stable colorectal cancer. Oncogene 23: 617-628, 2004

33. Wooster1 R, Cornwell W, Jing J, et al: Models for response to the MEK inhibitor GSK1120212 confirm RAS and BRAF mutations as predictive biomarkers and suggest other, unexpected tumor types for clinical evaluation. Eur J Cancer (Suppl) 6: S33-S34, 2008.

34. Allen WL and Johnston PG. Role of genomic markers in colorectal cancer treatment. J Clin Oncol 23: 4545-4552, 2005. 